\title{
SPHERES IN INFINITE-DIMENSIONAL NORMED SPACES ARE LIPSCHITZ CONTRACTIBLE
}

\author{
Y. BENYAMINI ${ }^{1}$ AND Y. STERNFELD
}

\begin{abstract}
Let $X$ be an infinite-dimensional normed space. We prove the following:
(i) The unit sphere $\{x \in X:\|x\|=1\}$ is Lipschitz contractible.

(ii) There is a Lipschitz retraction from the unit ball of $X$ onto the unit sphere.

(iii) There is a Lipschitz map $T$ of the unit ball into itself without an approximate fixed point, i.e. $\inf \{\|x-T x\|:\|x\| \leqslant 1\}>0$.
\end{abstract}

Introduction. Let $X$ be a normed space, and let $B_{X}=\{x \in X:\|x\| \leqslant 1\}$ and $S_{X}=\{x \in X:\|x\|=1\}$ be its unit ball and unit sphere, respectively.

Brouwer's fixed point theorem states that when $X$ is finite dimensional, every continuous self-map of $B_{X}$ admits a fixed point. Two equivalent formulations of this theorem are the following.

1. There is no continuous retraction from $B_{X}$ onto $S_{X}$.

2. $S_{X}$ is not contractible, i.e., the identity map on $S_{X}$ is not homotopic to a constant map.

It is well known that none of these three theorems hold in infinite-dimensional spaces (see e.g. [1]). The natural generalization to infinite-dimensional spaces, however, would seem to require the maps to be uniformly-continuous and not merely continuous. Indeed in the finite-dimensional case this condition is automatically satisfied.

In this article we show that the above three theorems fail, in the infinite-dimensional case, even under the strongest uniform-continuity condition, namely, for maps satisfying a Lipschitz condition. More precisely, we prove

THEOREM. Let $X$ be an infinite-dimensional normed space. Then

(1) The unit sphere $S_{X}$ is Lipschitz contractible.

(2) There is a Lipschitz retraction from $B_{X}$ onto $S_{X}$.

(3) There is a Lipschitz map $T: B_{X} \rightarrow B_{X}$ without an approximate fixed point, i.e. $\inf \left\{\|x-T x\|: x \in B_{X}\right\}=d>0$.

The first study of Lipschitz maps without approximate fixed points, and Lipschitz retractions from $B_{X}$ onto $S_{X}$, was done by K. Goebel [3]. B. Nowak [5] proved the theorem for several classical Banach spaces. Our work was greatly influenced by the work of Nowak. Actually, the general scheme of the proof as well as two of the three

Received by the editors November 1, 1982.

1980 Mathematics Subject Classification. Primary 47H10, 46B20.

${ }^{1}$ The first author was partially supported by the Fund for the Promotion of Research at the Technion - Israel Institute of Technology. 
main steps in the proof (Definition 1 and Propositions 1 and 3) are slight modifications of results in [5] which we reproduce for the sake of completeness and because of the many misprints in [5].

In [4] the authors study fixed point properties of mappings whose iterates satisfy a uniform Lipschitz condition. In this respect it is interesting to note that the map constructed in part (3) of the Theorem has this property (as follows immediately from its definition in the next paragraph).

Note that parts (2) and (3) of the Theorem follow immediately from (1). Indeed if $H(t, x)$ is a Lipschitz homotopy joining the constant $x_{0} \in S_{X}$ to the identity on $S_{X}$, then

$$
r(x)= \begin{cases}H(2\|x\|-1, x /\|x\|), & 1 / 2 \leqslant\|x\| \leqslant 1, \\ x_{0}, & 0 \leqslant\|x\| \leqslant 1 / 2\end{cases}
$$

is a Lipschitz retraction from $B_{X}$ onto $S_{X}$. It is also easy to check that the map $f(x)=-r(x)$ is then a Lipschitz self-map of $B_{X}$ without an approximate fixed point.

In the next section we shall formulate three propositions and deduce the theorem from them. These three propositions will be proved in $\S \S 2-4$, respectively.

We use standard terminology and notation. The reader is referred to [2] for basic facts on normed spaces.

We end this introduction with a very useful observation which we shall use later without further notice. If \|\|$_{1}$ and \|\|$_{2}$ are two equivalent norms on a linear space $X$, then the pair $\left(B_{1}, S_{1}\right)$ is Lipschitz equivalent to the pair $\left(B_{2}, S_{2}\right)$ under the map $x \rightarrow\|x\|_{1} x /\|x\|_{2}(0 \rightarrow 0)$. (Here $B_{i}$ (resp. $S_{i}$ ) is the unit ball (resp. sphere) of $X$ with respect to the norm \|\|$_{i}, i=1,2$.) It follows that any Lipschitz property of $B_{X}$ and $S_{X}$-and, in particular, our Theorem-can be proved under any norm equivalent to the original given norm.

1. In this section we give a definition and three propositions and then deduce the Theorem. The propositions will be proved in the subsequent sections.

Definition. Let $(S, d)$ be a metric space, $y_{0} \in S$ and $\varepsilon>0$. The point $y_{0}$ is said to be an $\varepsilon$-escaping point if there exists a Lipschitz mapping $T: S \rightarrow S$ satisfying:

(1.1) $T$ is Lipschitz homotopic to the identity on $S$.

(1.2) $\inf \left\{d\left(T^{n} y_{0}, T^{m} y_{0}\right): n>m \geqslant 0\right\} \geqslant 5 \varepsilon$.

(1.3) For all $n \geqslant 0, T$ maps $B_{S}\left(T^{n} y_{0}, \varepsilon\right)$ isometrically onto $B_{S}\left(T^{n+1} y_{0}, \varepsilon\right)$ (where $\left.B_{S}(y, \varepsilon)=\{x \in S: d(x, y) \leqslant \varepsilon\}\right)$.

(1.4) For all $n \geqslant 0, T^{-1}\left(B_{S}\left(T^{n+1} y_{0}, \varepsilon\right)\right)=B_{S}\left(T^{n} y_{0}, \varepsilon\right)$.

Proposition 1. Let $y_{0}$ be an e-escaping point in a metric space $S$, and let $Z$ be another metric space. Let $g:[-1,1] \times S \rightarrow Z$ be a Lipschitz map which constantly attains the value $z_{0} \in Z$ outside the set $\left[\frac{1}{4}, \frac{3}{4}\right] \times B_{S}\left(y_{0}, \varepsilon\right)$. Then $g$ is Lipschitz homotopic to the constant function $z_{0}$ in $[-1,1] \times S$ by a Lipschitz homotopy $H_{\tau}(t, x)$ $(0 \leqslant \tau \leqslant 1,(t, x) \in[-1,1] \times S)$, for which $H_{\tau}(t, x)=z_{0}$ whenever $|t| \geqslant \frac{3}{4}$.

REMARK. The fact that $g$ is Lipschitz homotopic to $z_{0}$ is, of course, obvious and does not require any assumptions on $y_{0}$. Indeed, the homotopy $h_{\tau}(t, x)=g(t \tau, x)$ does the job. The assumption that $y_{0}$ is $\varepsilon$-escaping is used to construct a homotopy $H_{\tau}$ with the additional property that $H_{\tau}(t, x)=z_{0}$ whenever $|t| \geqslant \frac{3}{4}$. 
Proposition 2. Let $X$ be an infinite-dimensional normed space and $\varepsilon \leqslant 1 / 500$. Then $X$ admits an equivalent norm with respect to which $S_{X}$ has an e-escaping point.

Proposition 3. Let $X$ be a normed space, and let $x_{0} \in S_{X}$ and $\varepsilon>0$. Then the identity map on $S_{X}$ is Lipschitz homotopic to a mapping $f: S_{X} \rightarrow S_{X}$, which constantly attains the value $-x_{0}$ outside the set $\left\{x \in S_{X}:\left\|x-x_{0}\right\|<\varepsilon\right\}$.

Proof of The Theorem. Let $X$ be an infinite-dimensional normed space, and let $Y$ be a closed subspace of $X$ of codimension one. By Proposition 2 there is an equivalent norm ||| ||| on $Y$ so that the unit sphere $S_{Y}$ with respect to this new norm admits an $\varepsilon$-escaping point, $y_{0}$, for some $\varepsilon \leqslant 1 / 500$. We now identify $X$ with $R \oplus Y$ under the norm $\|(t, y)\|=\max (\|y\|,|t|)$. This gives a norm on $X$, equivalent to the original one, and we shall prove the Theorem for this norm. To save notation we assume this is the given norm on $X$, and we then have $S_{X}=\left([-1,1] \times S_{Y}\right) \cup$ $\left(\{-1,1\} \times B_{Y}\right)$.

Set $x_{0}=\left(\frac{1}{2}, y_{0}\right) \in S_{X}$ and $z_{0}=-x_{0}$. By Proposition 3 there is a Lipschitz map $f$ : $S_{X} \rightarrow S_{X}$. Lipschitz homotopic to the identity on $S_{X}$, so that $f(x)=z_{0}=-x_{0}$ whenever $\left\|x-x_{0}\right\| \geqslant \varepsilon$. If $x=(t, y) \in S_{X}$ satisfies $\left\|x-x_{0}\right\|<\varepsilon$, then, since $\varepsilon<\frac{1}{4}$, $(t, y) \in\left[\frac{1}{4}, \frac{3}{4}\right] \times B_{S_{Y}}\left(y_{0}, \varepsilon\right) \subset[-1,1] \times S_{Y}$. It follows that $g=f_{\left[[-1,1] \times S_{Y}\right.}$ satisfies the conditions of Proposition 1 (with $S=S_{Y}$ ). As $y_{0}$ is an $\varepsilon$-escaping point in $S_{Y}$, it follows that $g$ is Lipschitz homotopic, as a map from $[-1,1] \times S_{Y}$ into $S_{X}$, to the constant $z_{0}=-x_{0} \in S_{X}$, by a homotopy $H_{\tau}(t, y)$ satisfying $H_{\tau}(t, y)=z_{0}$ whenever $|t| \geqslant \frac{3}{4}$.

Now extend $H_{\tau}$ to a homotopy $F_{\tau}$ in $S_{X}$ by defining $F_{\tau}(x)=z_{0}$ for $x \in S_{X} \backslash[-1,1]$ $\times S_{Y}$ and all $\tau$. It is easy to see that $F_{\tau}$ is a Lipschitz homotopy in $S_{X}$ joining $f$ to the constant $z_{0}$. Since $f$ is Lipschitz homotopic to the identity on $S_{X}$, it follows that $S_{X}$ is Lipschitz contractible.

Remarks. (1) The definition of $\varepsilon$-escaping point, Propositions 1 and 3 , and the general scheme of the proof are slight modifications of the results of Nowak [5], where the same terminology is also used. Notice, however, that our definition of an $\varepsilon$-escaping point is more general than the one used in [5].

(2) Analyzing the proof of the Theorem and the propositions, one can see that there is, in fact, an absolute constant $K<\infty$, independent of the given infinite-dimensional $X$, so that the identity on $S_{X}$ is contractible to a constant map by a homotopy satisfying a Lipschitz condition with constant at most $K$. To see this one should only check that all the renormings in the proofs can be made up to some absolute constants, and that $\varepsilon$ can be chosen independent of $X$. A similar remark holds concerning (2) and (3) of the Theorem. We leave the details to the interested reader.

2. Proof of Proposition 1. Let $T$ be the map associated to $y_{0}$ by the definition of an $\varepsilon$-escaping point. Define two maps $f_{i}:[-1,1] \times S \rightarrow Z, i=0,1$, by

$$
f_{0}(t, x)= \begin{cases}g\left(t, T^{-n} x\right), & t \geqslant 0 \text { and } x \in B_{S}\left(T^{n} y_{0}, \varepsilon\right), n \geqslant 0, \\ g\left(-t, T^{-n} x\right), & t \leqslant 0 \text { and } x \in B_{S}\left(T^{n} y_{0}, \varepsilon\right), n \geqslant 1, \\ z_{0}, & \text { otherwise; }\end{cases}
$$




$$
f_{1}(t, x)= \begin{cases}g\left(|t|, T^{-n} x\right), & x \in B_{S}\left(T^{n} y_{0}, \varepsilon\right), n \geqslant 0, \\ z_{0}, & \text { otherwise. }\end{cases}
$$

The following figure illustrates the nature of $g, f_{0}$ and $f_{1}$ :
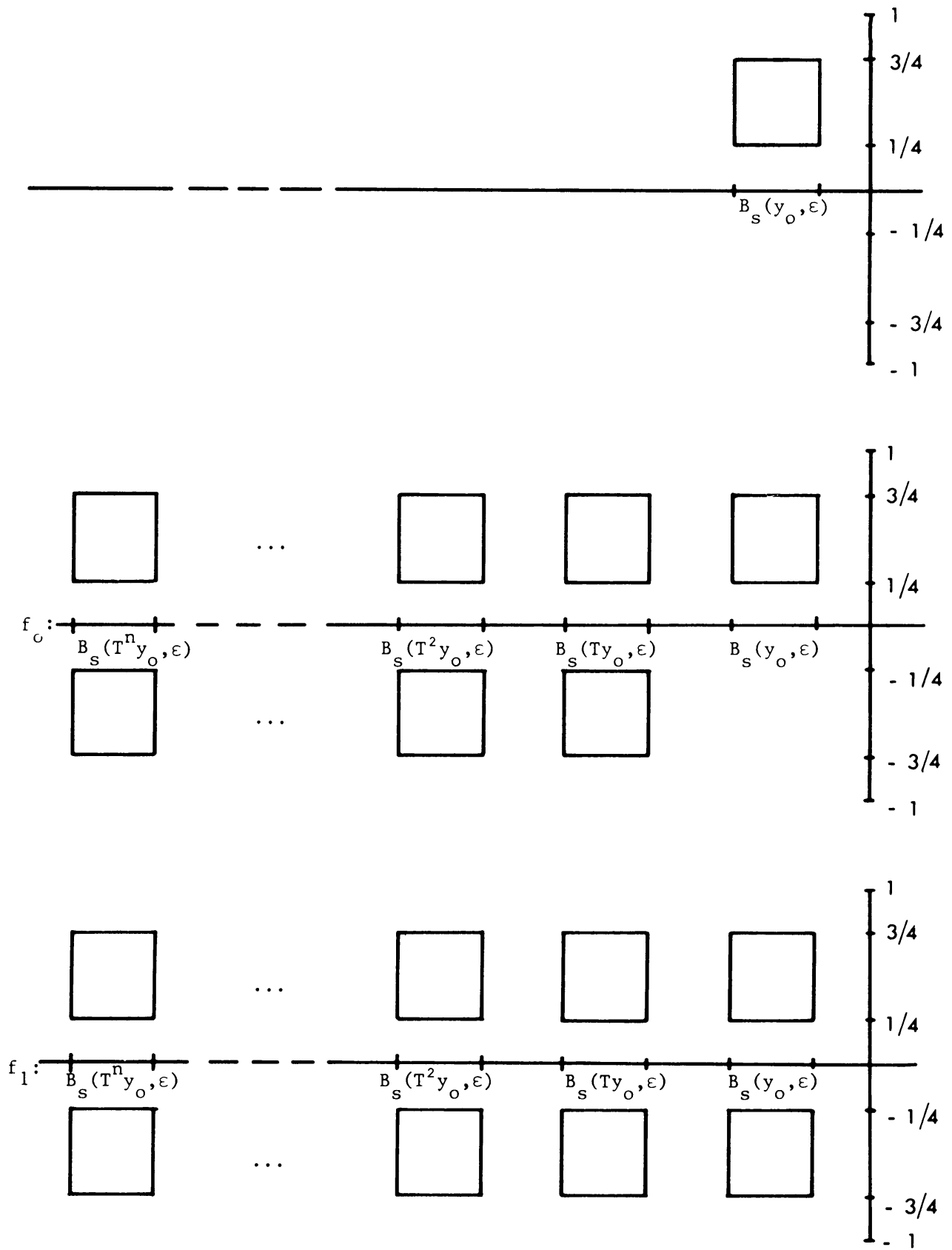

Here $S$ is realized as the horizontal line, the maps are constant (and equal to $z_{0}$ ) outside the "rectangles", and inside each "rectangle" they are defined by the 
corresponding value (with respect to $\left.T^{-n}\right)$ of $g$ in the "rectangle" $\left[\frac{1}{4}, \frac{3}{4}\right] \times B_{S}\left(y_{0}, \varepsilon\right)$. Note that by the definition of an $\varepsilon$-escaping point, the "rectangles" are indeed disjoint (in fact the distance between two "rectangles" is at least $3 \varepsilon$ ), and also that $T^{-n}$ is an isometry of $B_{S}\left(T^{n} y_{0}, \varepsilon\right)$ onto $B_{S}\left(y_{0}, \varepsilon\right)$. Thus $f_{i}$ are indeed Lipschitz maps. By (1.1) $T$ is Lipschitz homotopic to the identity, and let $G_{\tau}$ be the Lipschitz homotopy, in $S$, joining the identity to $T$. Then

$$
F_{\tau}(t, x)= \begin{cases}f_{0}(t, x), & t \geqslant 0 \\ f_{0}\left(t, G_{\tau}(x)\right), & t \leqslant 0\end{cases}
$$

is a Lipschitz homotopy in $[-1,1] \times S$ joining $f_{0}$ to $f_{1}$.

The map $F_{\tau}^{1}(t, x)=f_{1}(|t|(1-\tau)+\tau, x)$ gives a Lipschitz homotopy joining $f_{1}$ to the constant $z_{0}$, and the map

$$
F_{\tau}^{0}(t, x)= \begin{cases}f_{0}(|t| \tau+(1-\tau), x), & x \notin B_{S}\left(y_{0}, \varepsilon\right), \\ g(t, x), & x \in B_{S}\left(y_{0}, \varepsilon\right),\end{cases}
$$

is a Lipschitz homotopy joining $g$ to $f_{0}$. The desired homotopy $H_{\tau}$ is now obtained by applying successively $F_{\tau}^{0}, F_{\tau}$ and $F_{\tau}^{1}$. Since all three have the constant value $z_{0}$ for $|t| \geqslant \frac{3}{4}$, the same holds for $H_{\tau}$.

3. To prove Proposition 2, we shall need two lemmas.

LEMMA 1. Let $X$ be a normed space and $\frac{1}{8}>\varepsilon>0$. Let $a, b$ be two points in $X$ so that $a \neq b$ and $\|a\|=\|b\|=\frac{1}{4}$. There exists a map $U=U_{a, b}: B_{X} \rightarrow B_{X}$ satisfying:

(3.1) $U$ satisfies a Lipschitz condition with constant at most $1+1 / 2 \varepsilon$.

(3.2) $U$ maps $B_{X}(a, \varepsilon)$ isometrically onto $B_{X}(b, \varepsilon)$, and $U a=b$.

(3.3) $U^{-1}\left(B_{X}(b, \varepsilon)\right)=B_{X}(a, \varepsilon)$.

(3.4) $U x=x$ whenever $d(x,[a, b]) \geqslant 2 \varepsilon$ (where $[a, b]=\{t a+(1-t) b: 0 \leqslant t \leqslant$ 1)). In particular $U x=x$ for $x \in S_{X}$.

(3.5) $U$ maps lines parallel to $[a, b]$ into themselves.

Proof. Define

$$
\alpha(x)= \begin{cases}1, & d(x,[a, b]) \leqslant \varepsilon, \\ 2-\varepsilon^{-1} d(x,[a, b]), & \varepsilon \leqslant d(x,[a, b]) \leqslant 2 \varepsilon, \\ 0, & d(x,[a, b])>2 \varepsilon,\end{cases}
$$

Then $\alpha: B_{X} \rightarrow[0,1]$ satisfies a Lipschitz condition with constant $1 / \varepsilon$.

Now set $U x=x+\alpha(x)(b-a)$. The conditions on $\|a\|,\|b\|$ and $\varepsilon$ immediately imply that $U$ maps $B_{X}$ into itself, and that it satisfies (3.1)-(3.5). We only check (3.3): If $U x \in B(b, \varepsilon)$, we have

$$
\varepsilon \geqslant\|b-U x\|=\|b-x-\alpha(x)(b-a)\|=\|[\alpha(x) a+(1-\alpha(x)) b]-x\| .
$$

But $0 \leqslant \alpha(x) \leqslant 1$, so that $\alpha(x) a+(1-\alpha(x)) b \in[a, b]$. Thus $d(x,[a, b]) \leqslant \varepsilon$ and $\alpha(x)=1$, i.e. $\varepsilon \geqslant\|b-U x\|=\|b-x-(b-a)\|=\|a-x\|$, and $x \in B(a, \varepsilon)$. 
Lemma 2. Let $X$ be an infinite-dimensional normed space and $0<\varepsilon \leqslant 1 / 500$. Then there exists a point $x_{0} \in B_{X}$ which is an $\varepsilon$-escaping point in $B_{X}$ with respect to a map $T$ : $B_{X} \rightarrow B_{X}$ which, in addition to (1.1)-(1.4), also satisfies

$$
T x=x \quad \text { for } x \in S_{X} .
$$

Proof. Note that the homotopy condition (1.1) is trivially satisfied here because $B_{X}$ is convex, hence Lipschitz contractible. Let $\left\{w_{n}\right\}_{n=1}^{\infty}$ be a normalized basic sequence in $X$ with biorthogonal functionals $\left\{\varphi_{n}\right\} \subset X^{*}$ satisfying $\left\|\varphi_{n}\right\| \leqslant 4$, and set $z_{n}=w_{n} / 4$. (See [2, p. 93].)

Denote by $L_{n, k}(n \neq k)$ the straight line $\left\{t z_{n}+(1-t) z_{k}: t \in R\right\}$ passing through $z_{n}$ and $z_{k}$. If $\{n, k\} \cap\{m, l\}=\varnothing$, then

$$
d\left(L_{n, k}, L_{m, l}\right) \geqslant 1 / 32>10 \varepsilon
$$

Indeed, if $x=t z_{n}+(1-t) z_{k}, \quad y=\zeta z_{m}+(1-\zeta) z_{l}$, assume $|t| \geqslant \frac{1}{2}$ (otherwise $|1-t| \geqslant \frac{1}{2}$, and then

$$
\|x-y\| \geqslant \frac{1}{4}\left|\varphi_{n}\left(t z_{n}+(1-t) z_{k}-\zeta z_{m}-(1-\zeta) z_{l}\right)\right| \geqslant|t| / 16 \geqslant 1 / 32 .
$$

Denote by $U_{n, m}$ the map constructed in Lemma 1 for $a=z_{n}, b=z_{m}$ and the given $\varepsilon$. Note that by (3.7), if $\{r, k\} \cap\{m, l\}=\varnothing$, then $U_{n, k}(x)=x$ whenever $d\left(x, L_{m, l}\right)$ $\leqslant 2 \varepsilon$, and, in particular, when $U_{m, l}(x) \neq x$, or $x=U_{m, l}(y)$ for some $y \neq x$. Thus the infinite composition $V_{1}(x)=\left(\cdots \circ U_{2 n-1,2 n} \circ \cdots \circ U_{3,4} \circ U_{1,2}\right)(x)$ is well defined and satisfies:

(3.8) $V_{1}$ is a Lipschitz map with constant at most $1+1 / 2 \varepsilon$.

(3.9) $V_{1}$ maps $B_{X}\left(z_{2 n-1}, \varepsilon\right)$ isometrically onto $B_{X}\left(z_{2 n}, \varepsilon\right)$.

(3.10) $V_{1}^{-1}\left(B_{X}\left(z_{2 n}, \varepsilon\right)\right)=B_{X}\left(z_{2 n-1}, \varepsilon\right)$.

(3.11) $V_{1} x=x$ for $x \in S_{X}$.

Defining similarly $V_{2}(X)=\left(\cdots \circ U_{2 n, 2 n+1} \circ \cdots \circ U_{4.5} \circ U_{2.3}\right)(x), V_{2}$ also satisfies (3.8), (3.11) and:

(3.12) $V_{2}$ maps $B_{X}\left(z_{2 n}, \varepsilon\right)$ isometrically onto $B_{X}\left(z_{2 n+1}, \varepsilon\right)$.

(3.13) $V_{2}^{-1}\left(B_{X}\left(z_{2 n+1}, \varepsilon\right)\right)=B_{X}\left(z_{2 n}, \varepsilon\right)$.

We now define $T=V_{2} V_{1}$. T is a Lipschitz function, and $z_{1}$ is an $\varepsilon$-escaping point with an associated map $T$. Indeed, as observed before, (1.1) is trivially satisfied. Also $T^{n} z_{1}=z_{2 n+1}$, and (1.2) follows from (3.7). Condition (1.3) follows from (3.9) and (3.12), and (1.4) from (3.10) and (3.13).

Proof of Proposition 2. Let $Y$ be a closed subspace of $X$ of codimension one. Identify $X$ with $R \oplus Y$ and, by equivalently renorming $X$, if necessary, assume that $\|(t, y)\|=\max (|t|,\|y\|)$.

By Lemma 2 there is a point $y_{0} \in B_{Y}$ which is $\varepsilon$-escaping in $B_{Y}$ with respect to a Lipschitz map $V: B_{Y} \rightarrow B_{Y}$ satisfying $V y=y$ whenever $\|y\|=1$.

We have $S_{X}=\left(\{1\} \times B_{Y}\right) \cup\left([-1,1] \times S_{Y}\right) \cup\left(\{-1\} \times B_{Y}\right)$, and define $T: S_{X} \rightarrow$ $S_{X}$ by

$$
T(t, y)= \begin{cases}(1, V y), & t=1 \\ (t, y), & t \neq 1\end{cases}
$$


Then $T$ is a well-defined Lipschitz map, $T: S_{X} \rightarrow S_{X}$, and $\left(1, y_{0}\right)$ is an $\varepsilon$-escaping point of $S_{X}$ associated with this $T$. Indeed (1.2)-(1.4) follow immediately from the corresponding properties of $V$, and $T$ is Lipschitz homotopic to the identity by the homotopy $H_{\tau}(x)=\tau T x+(1-\tau) x$. By the special structure of $S_{X}$ and the fact that $T x \neq x$ only for points $x=(t, y)$ with $t=1, y \in B_{Y}$, it follows that $H_{\tau}(x)$ is indeed a point of $S_{X}$ whenever $x$ is.

4. Proof of Proposition 3. The proposition is trivial when $X$ is one dimensional, so assume $\operatorname{dim} X \geqslant 2$. Fix $x_{0} \in S_{X}$ and let $\varphi \in X^{*}$ satisfy $\|\varphi\|=\varphi(x)=1$. Renorming $X$ by $\|x\|=|\varphi(x)|+\left\|x-\varphi(x) x_{0}\right\|$, we obtain a representation of $X$ as $R \oplus Y$, with $Y=\operatorname{Ker}(\varphi)$, and with norm $\|(t, y)\|=|t|+\|y\|$. The point $x_{0}$ is identified with $(1,0)$. To save notation we assume this is the given norm on $X$.

Now define, for $\varepsilon / 2 \leqslant \tau \leqslant 2$, a function $\varphi_{\tau}:[-1,1] \rightarrow[-1,1]$ by

$$
\varphi_{\tau}(t)= \begin{cases}2 \tau^{-1} t+1-2 \tau^{-1}, & 1-\tau \leqslant t \leqslant 1 \\ -1, & -1 \leqslant t \leqslant 1-\tau\end{cases}
$$

and let

$$
F_{\tau}(t, y)=\left(\varphi_{\tau}(t), \frac{1-\left|\varphi_{\tau}(t)\right|}{1-|t|} y\right) \quad\left(F_{\tau}( \pm 1,0)=( \pm 1,0)\right) .
$$

If $(t, y) \in S_{X}$, i.e. $|t|+\|y\|=1$, then also $F_{\tau}(t, y) \in S_{X}$, and $F_{\tau}, \varepsilon / 2 \leqslant \tau \leqslant 2$, is a Lipschitz homotopy of $S_{X}$, with Lipschitz constant $c / \varepsilon$ for some $c<\infty$. (We leave the straightforward verification to the reader.)

For $\tau=2, F_{2}(t, y)=(t, y)$, i.e. $F_{2}$ is the identity, while $f(t, y)=F_{\varepsilon / 2}(t, y)$ satisfies $f(x)=(-1,0)=-x_{0}$ whenever $\left\|x-x_{0}\right\| \geqslant \varepsilon$.

\section{REFERENCES}

1. C. Bessaga and A. Pelczyński, Selected topics in infinite dimensional topologv, PWN, Warsaw, 1975.

2. M. M. Day, Normed linear spaces, 3rd ed., Springer-Verlag, Berlin, 1973.

3. K. Goebel, On the minimal displacement of points under Lipschitzian mappings, Pacific J. Math. 45 (1973), 151-163.

4. K. Goebel and W. A. Kirk, A fixed point theorem for transformations whose iterations have uniform Lipschitz constant, Studia Math. 47 (1973), 135-140.

5. B. Nowak, On the Lipschitzian retraction of the unit ball in infinite dimensional Banach spaces onto its boundary, Bull. Acad. Polon. Sci. Sér. Sci. Math. 27 (1979), 861-864.

Department of Mathematics, Technion-IsRael Institute of Technology, Haifa, 32000 IsRael

Department of Mathematics, University of Haifa, Mount Carmel, Haifa, Israel 